
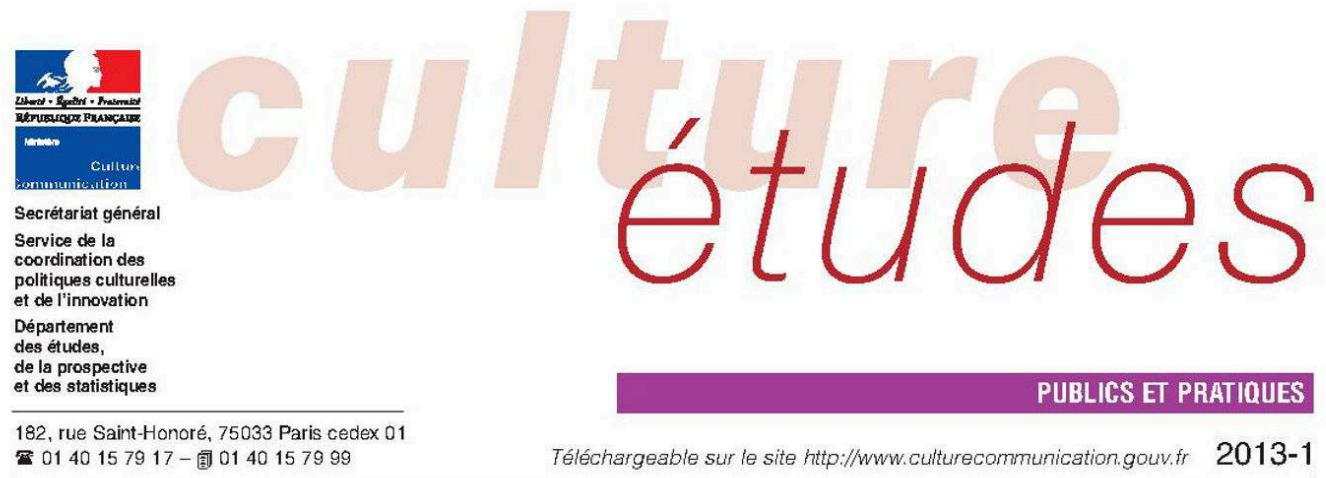

PUBLIGS ET PRATIQUES

番 0140157917 - 国 0140157999

Téléchargeable sur le site http://www.culturecommunication. gouv.fr

2013-1

\title{
Sociabilités et sortie au théâtre
}

\section{Dominique Pasquier*}

\section{Sociability and Theatre}

Pour le public, le théâtre est avant tout une expérience culturelle, la rencontre avec une ouvre, et un contact physique avec des comédiens. Faut-il pour autant négliger les dimensions sociales de cette expérience ? On peut appréhender cette question de plusieurs manières : en s'intéressant aux échanges que suscite la pratique (avec qui on en parle?), ou en analysant les réseaux de sociabilité dans lesquels elle s'insère (avec qui on y va ${ }^{1}$ ?). Il ne s'agit donc pas d'identifier les caractéristiques générales des spectateurs de théâtre ni d'élaborer une typologie des spectateurs, comme ont pu le faire d'autres recherches ${ }^{2}$, mais d'étudier les ressources sociales qui sont nécessaires pour entrer dans la pratique et la stabiliser. L'entreprise ne se veut ni irrévérencieuse à l'égard du rapport aux ouvres (qu'il ne s'agit pas de nier), ni relativiste quant à l'existence de hiérarchies culturelles complexes selon les salles et les répertoires. Il s'agit simplement d'éclaircir la part du social dans ce type de pratique culturelle en s'appuyant sur les résultats d'une exploitation secondaire de la dernière enquête Pratiques culturelles des Français et d'une série d'entretiens menés auprès de spectateurs de théâtre (voir Éléments de méthodologie, p. 10).

\section{AUPRÈs DE QUI S'INFORMER SUR LES PIÈCES ?}

Faible influence de la prescription médiatique

Un premier constat s'impose : le théâtre est un marché peu équipé en termes de dispositifs d'aide au choix par rapport à d'autres secteurs culturels. Si l'on reprend les dispositifs de jugement recensés par Lucien Karpik ${ }^{3}$, force est de constater qu'il n'y a pas d'appellations officielles, que les prix sont rares (les récompenses des Molière remontent à quelques années seulement et ont de toute façon très peu d'influence sur les choix des publics) et que les techniques de diffusion de l'information sont assez simples : des affiches dans des lieux publics et des brochures distribuées par les théâtres.

Toutefois, dans ce contex te de pénurie d'information, le théâtre privé n'est pas logé à la même enseigne que le théâtre public, et le repárage dans l'offre est bien plus complexe dans les grandes villes (et particulièrement à Paris) qu'il ne l'est ailleurs. L'exemple de la couverture critique des pièces illustre bien ce phénomène.

\footnotetext{
* Dominique Pasquier est sociologue, directrice de recherche au CNRS. Plusieurs de ses recherches ont porté sur la question des liens entre culture et sociabilité.

1. Une troisième piste, celle de l'expérience sociale des spectateurs en situation de coprésence dans une même salle pour assister à une représentation, ne sera pas traitée ici. Elle est explorée dans le dernier chapitre du rapport en ligne, consultable sur www.culturecommunication.gouv.frßEtudes-etstatistiques, rubrique Publications.

2. J.-M. GUY, L. Mironer, les Publics du théâtre : fréquentation et image du théâtre dans la population française ágée de 15 ans et plus, Paris, Ministère de la Culture et de la Communication, DEP, La Documentation française, 1988 ;V. BeAudoujn, B. Maresca, les Publics de la Comédie-Française, Paris, Ministère de la Culture et de la Communication, DEP, La Documentation française, 1997; P. CIBO1s, «Les abonnés du théâtre : un public hétérogène », dans Olivier Donnat (sous la dir. de), Regards croisés sur les pratiques culturelles, Paris, Ministère de la Culture et de la Communication, DEP, La Documentation française, coll. «Questions de culture », 2003, p. 171-187; A. DJAKOUANE, les Carrières de spectateurs. Vers une sociologie des formes de prescription thêatrale, doctorat de sociologie sous la direction d'Emmanuel PEDLER, Paris, EHESS, 2007.
}

3. L. KARPIK, l'Économie des singularités, Paris, Gallimard, 2007. 


\section{Sociabilités et sortie au théâtre}

Sociability and Theatre

Dominique Pasquier

Éditeur : Département des études, de la prospective et des statistiques

Lieu d'édition : Paris

Année d'édition : 2013

Date de mise en ligne : 8 juillet 2015

Collection : Culture études

ISBN électronique : 9782111399082

\section{Q boooks}

http://books.openedition.org

\section{Édition imprimée}

Date de publication : 1 février 2013

Nombre de pages : 12

\section{Référence électronique}

PASQUIER, Dominique. Sociabilités et sortie au théâtre. Nouvelle édition [en ligne]. Paris : Département des études, de la prospective et des statistiques, 2013 (généré le 25 avril 2021). Disponible sur Internet : <http://books.openedition.org/deps/111>. ISBN : 9782111399082

(C) Département des études, de la prospective et des statistiques, 2013

Creative Commons - Attribution - Pas d'Utilisation Commerciale 3.0 non transposé - CC BY-NC 3.0 

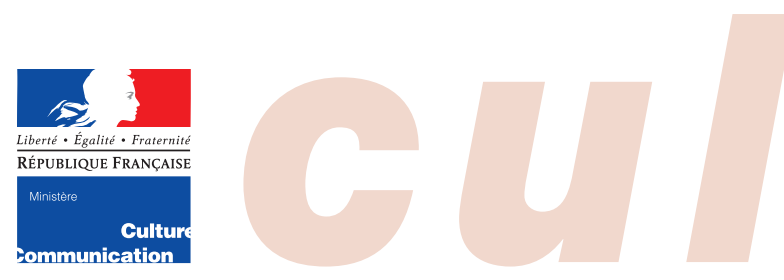

Secrétariat général

Service de la

coordination des

politiques culturelles

et de l'innovation

Département

des études,

de la prospective

et des statistiques

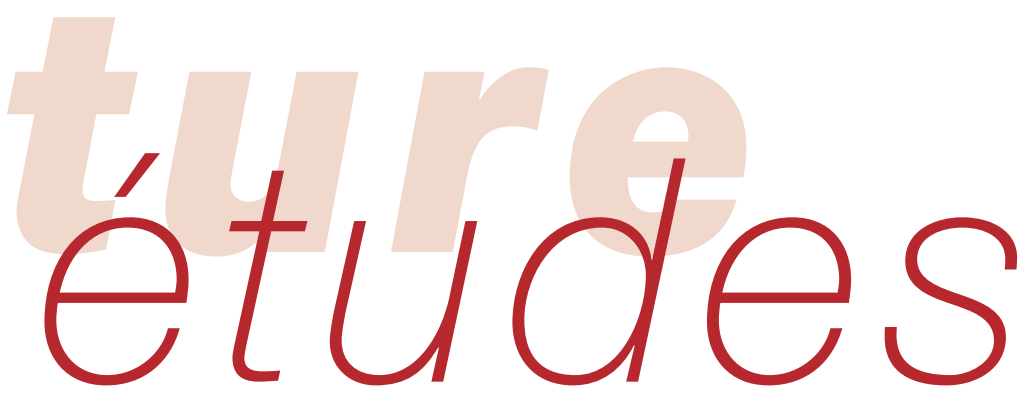

182, rue Saint-Honoré, 75033 Paris cedex 01

푤 0140157917 - 固 0140157999

PUBLICS ET PRATIQUES

\section{Sociabilités et sortie au théâtre}

\section{Dominique Pasquier*}

\section{Sociability and Theatre}

Pour le public, le théâtre est avant tout une expérience culturelle, la rencontre avec une œuvre, et un contact physique avec des comédiens. Faut-il pour autant négliger les dimensions sociales de cette expérience ? On peut appréhender cette question de plusieurs manières : en s'intéressant aux échanges que suscite la pratique (avec qui on en parle ?), ou en analysant les réseaux de sociabilité dans lesquels elle s'insère (avec qui on y va ${ }^{1}$ ?). Il ne s'agit donc pas d'identifier les caractéristiques générales des spectateurs de théâtre ni d'élaborer une typologie des spectateurs, comme ont pu le faire d'autres recherches ${ }^{2}$, mais d'étudier les ressources sociales qui sont nécessaires pour entrer dans la pratique et la stabiliser. L'entreprise ne se veut ni irrévérencieuse à l'égard du rapport aux œuvres (qu'il ne s'agit pas de nier), ni relativiste quant à l'existence de hiérarchies culturelles complexes selon les salles et les répertoires. Il s'agit simplement d'éclaircir la part du social dans ce type de pratique culturelle en s'appuyant sur les résultats d'une exploitation secondaire de la dernière enquête Pratiques culturelles des Français et d'une série d'entretiens menés auprès de spectateurs de théâtre (voir Éléments de méthodologie, p. 10).

\author{
AUPRÈS DE QUI S'INFORMER \\ SUR LES PIÈCES ?
}

\section{Faible influence de la prescription médiatique}

Un premier constat s'impose : le théâtre est un marché peu équipé en termes de dispositifs d'aide au choix par rapport à d'autres secteurs culturels. Si l'on reprend les dispositifs de jugement recensés par Lucien Karpik ${ }^{3}$, force est de constater qu'il n'y a pas d'appellations officielles, que les prix sont rares (les récompenses des Molière remontent à quelques années seulement et ont de toute façon très peu d'influence sur les choix des publics) et que les techniques de diffusion de l'information sont assez simples : des affiches dans des lieux publics et des brochures distribuées par les théâtres.

Toutefois, dans ce contexte de pénurie d'information, le théâtre privé n'est pas logé à la même enseigne que le théâtre public, et le repérage dans l'offre est bien plus complexe dans les grandes villes (et particulièrement à Paris) qu'il ne l'est ailleurs. L'exemple de la couverture critique des pièces illustre bien ce phénomène.

\footnotetext{
* Dominique Pasquier est sociologue, directrice de recherche au CNRS. Plusieurs de ses recherches ont porté sur la question des liens entre culture et sociabilité.

1. Une troisième piste, celle de l'expérience sociale des spectateurs en situation de coprésence dans une même salle pour assister à une représentation, ne sera pas traitée ici. Elle est explorée dans le dernier chapitre du rapport en ligne, consultable sur www.culturecommunication.gouv.fr/Etudes-etstatistiques, rubrique Publications.

2. J.-M. GuY, L. Mironer, les Publics du théâtre : fréquentation et image du théâtre dans la population française âgée de 15 ans et plus, Paris, Ministère de la Culture et de la Communication, DEP, La Documentation française, 1988 ; V. Beaudouin, B. MAResCA, les Publics de la Comédie-Française, Paris, Ministère de la Culture et de la Communication, DEP, La Documentation française, 1997 ; P. CiBoIs, «Les abonnés du théâtre : un public hétérogène », dans Olivier Donnat (sous la dir. de), Regards croisés sur les pratiques culturelles, Paris, Ministère de la Culture et de la Communication, DEP, La Documentation française, coll. «Questions de culture », 2003, p. 171-187; A. DJAKOUANE, les Carrières de spectateurs. Vers une sociologie des formes de prescription théâtrale, doctorat de sociologie sous la direction d'Emmanuel PEDLER, Paris, EHESS, 2007.

3. L. KARPIK, l'Économie des singularités, Paris, Gallimard, 2007.
} 
De manière générale, le rôle de la critique professionnelle est faible, beaucoup plus faible en tout cas que pour les livres ou les films de cinéma. C'est un constat déjà dressé par d'autres enquêtes sur le théâtre ${ }^{4}$, et qui mérite réflexion, surtout quand on se souvient du passé particulièrement riche de la critique théâtrale aux $\mathrm{XIX}^{\mathrm{e}}$ et $\mathrm{XX}^{\mathrm{e}}$ siècles. Les personnes interrogées déclarent faire un très faible usage des critiques et jugent la couverture de l'offre tardive, partielle, partiale, parfois même inutile. Il faut toutefois distinguer clairement le cas du théâtre privé et celui du théâtre subventionné.

Le décalage entre la couverture critique et l'offre, par exemple, concerne uniquement le théâtre subventionné et tient aux nouvelles stratégies de programmation des directeurs de salles. Des pièces qui, dans les années 1960 et 1970, étaient à l'affiche six à huit semaines d'affilée, sont aujourd'hui programmées au mieux deux ou trois semaines. Beaucoup de spectacles tournent en France mais ne sont joués que quelques soirées seulement dans chaque salle. L'augmentation considérable du nombre de spectacles au cours des dernières décennies s'est en effet accompagnée mécaniquement d'une baisse du nombre de représentations par spectacle. Il y a donc un problème de calendrier : la critique vient souvent trop tard. Évidemment trop tard pour les abonnés qui ont pris toutes leurs places avant le début de la saison, mais aussi trop tard par rapport à la durée des représentations : quand on lit une bonne critique et qu' on souhaite prendre des places, la pièce risque d'afficher complet, voire de ne plus être à l'affiche. À l'inverse, le théâtre privé prolonge ses spectacles en fonction de la demande du public et du succès des pièces, quitte à changer de salle et de comédiens, et bénéficie ainsi des effets du bouche-à-oreille et d'éventuelles couvertures par la presse audiovisuelle.

D'autre part, la critique de théâtre ne couvre qu'une partie de l'offre, c'est un fait, et la couvre d'une manière partiale, c'est une opinion exprimée par nombre de personnes interrogées. Dans les années 1960, Le Monde offrait quotidiennement des critiques de pièces - et listait même les salles et les horaires de l'offre parisienne ${ }^{5}$. Depuis, la signalisation de l'offre théâtrale a considérablement baissé au profit des programmes de cinéma et de télévision, et ce même dans les titres de presse écrite lus par les milieux culturellement favorisés. La télévision, quant à elle, pratique une « critique de célébration » : aux heures de grande écoute, elle couvre les pièces à gros budgets (comédies musicales, grandes reconstitutions historiques), ou les pièces à succès du théâtre privé6. L'approche est souvent centrée sur les comédiens, et plus proche de la promotion que de la critique argumentative.

La couverture très partielle de l'offre la rend plus dépendante des coups de cœur des journalistes. Ces parti pris ne sont pas du goût de tous les spectateurs, qui reprochent aux critiques de ne pas être fiables dans leurs jugements, d'intellectualiser les démarches, voire d'être de mauvaise foi par copinage. Les spectateurs assidus sont souvent les plus hostiles, comme s'ils déniaient aux critiques le monopole du jugement sur une pièce. «La critique c'est moi qui me la ferai ! » déclare un spectateur interrogé. Plus largement, force est de constater que les spectateurs, même s'ils ne nourrissent pas d'animosité particulière vis-à-vis des critiques de théâtre, s'y intéressent très peu et en tout cas n'en font jamais un guide pour le choix des pièces. Aucun des quatre-vingts spectateurs de théâtre interrogés n'a déclaré suivre régulièrement la couverture de l'offre théâtrale dans un titre de presse, aucun non plus n'a cité le nom d'un journaliste qui aurait une influence sur ses choix. Le divorce est sans doute profond.

En revanche, du côté des pièces du théâtre privé, les avis des internautes postés sur les sites de vente de billets à prix discount semblent jouer un certain rôle. Ces commentaires sont souvent courts et relèvent surtout d'impressions subjectives : on est à mille lieux du travail de la critique experte avec ses remises en perspective de l'œuvre, ses informations sur le sens donné par le créateur à son travail ou ses analyses du spectacle. Le système de notation par des étoiles - dont le nombre croît avec le plaisir éprouvé - peut éventuellement signaler des pièces meilleures que d'autres, mais les mauvaises notes sont en réalité fort rares. Pourtant, ces critiques sans appareillage critique semblent agir comme une aide au choix, surtout pour les spectateurs occasionnels. La présence de comédiens connus et l'appartenance au genre de la comédie de mœurs constituent aussi des points d'appui.

Dans le secteur subventionné, où les créations sont nombreuses et les repères moins balisés, les spectateurs s'appuient en partie sur les habitudes de programmation des salles qu'ils fréquentent et sur les conseils des services de relation au public. Ces professionnels ont désormais une action très différente de celle qui était la leur par le passé. Le poids des comités d'entreprise ayant radicalement baissé dans la commande de places, ces services agissent désormais de façon ciblée et personnalisée auprès de spectateurs «relais » qui agissent à leur tour auprès de leur entourage. Les groupes d'amis font partie de ce nouveau contexte.

\footnotetext{
4. Enquête sur le public de la Comédie-Française : les spectateurs de la salle Richelieu ont eu connaissance de la pièce à laquelle ils ont assisté par les brochures d'abonnement (45\%), par les journaux d'annonce des spectacles (15\%), par l'affichage extérieur (14\%), par leur entourage familial et amical (14\%), et par les critiques dans la presse pour $12 \%$ d'entre eux seulement - mais $37 \%$ ont lu ou entendu des critiques dans les médias avant d'aller voir cette pièce (voir V. Beaudouin, B. MAREsCA, les Publics de la Comédie-Française, op. cit., p. 167).

5. D. PAsquIER, "When the Elite Press Meets the Rise of Commercial Culture", dans J. GrIPSRUd et L. WeIBULl, Media, markets and Public Spheres. European Media at the Crossroads, Chicago, Intellect, 2009.

6. Aujourd'hui, il reste trois émissions spécialisées sur des stations de radio publiques (les Mercredis du théâtre sur France Culture, Studio théâtre et le Masque et la plume sur France Inter) contre plus d'une dizaine dans les années 1970 et 1980, tandis que les derniers magazines culturels ont été récemment déprogrammés des chaînes de France Télévision. Voir le Théâtre dans les fonds de l'Inathèque de France, document multigraphié, INA, décembre 2008 .
} 


\section{La prescription du réseau amical}

Les conseils de l'entourage ou la circulation de l'information par le bouche-à-oreille fonctionnent de façon particulière pour le théâtre. Comme c'est une pratique relativement peu répandue - en tout cas nettement moins que la lecture de livres ou la fréquentation du cinéma la probabilité d'entendre parler d'une pièce est faible à l'exception peut-être du milieu enseignant ${ }^{7}$. Pis, la pratique a une image élitiste. Comme le notait Erickson dans son enquête sur les conversations à propos de la culture sur le lieu de travail, le fait d'en parler avec des non-pratiquants risque fort d'être assimilé à un rituel de domination culturelle ${ }^{8}$. Les monographies de publics de théâtre soulignent d'ailleurs le poids relativement faible des conseils de l'entourage? .

Pourtant, même s'il ne fonctionne pas de façon horizontale et virale, comme c'est le cas pour le cinéma, le réseau joue un rôle. Il s'organise de façon pyramidale, autour d'un petit nombre de spectateurs qui assument, avec plus ou moins de bonheur et de réussite, le rôle de conseiller auprès de leur entourage. Certains spectateurs entraînent d'autres spectateurs. Cela peut se jouer à l'échelle d'un couple comme de groupes bien plus étendus. Ces individus ont en commun de toujours bénéficier d'une confiance fondée sur des expériences passées. Il n'y a pas de «bonnes » pièces, il y a des pièces plus ou moins adaptées au type d'expérience théâtrale que recherche un spectateur. Un conseiller doit donc connaître très bien les goûts et les attentes de ceux qu'il conseille. Et ces derniers ne peuvent lui faire confiance que si les conseils passés ont mené à des expériences satisfaisantes. La relation de conseil n'est jamais donnée d'emblée au nom d'une compétence supérieure, elle est relative et se crée dans la durée. Les conseillers occupent une place particulière dans la cartographie des liens : ce ne sont pas forcément les amis les plus proches mais ce sont des amis avec lesquels existe une intimité spécifique sur le théâtre, des personnes avec lesquelles on sait partager les mêmes goûts à un moment donné.

\section{La prise de risque dans le conseil sur les pièces}

Avec l'entourage, le conseil est donc prudent: pas avec tout le monde et pas pour des pièces dont on n'est pas sûr. Il faut dire que conseiller une pièce est bien plus compromettant que conseiller un livre ou même un film, car la sortie au théâtre est infiniment plus difficile à organiser. La plupart des personnes interrogées qui ont été placées dans un rôle de conseiller ou d'entrepreneur d'une sortie disent avoir vécu des expériences mitigées. ( «C'est une histoire à se faire charrier pendant des années! Si une pièce est vraiment mauvaise on vous en reparlera pendant des années !») On peut y perdre des amis ou du crédit social.

Les entretiens ont permis de montrer que tous les amateurs de théâtre ne se lancent pas dans le conseil de la même façon, même s'il semble que beaucoup aient envie de faire partager leur goût pour le théâtre. Certains sont prudents : ils testent les goûts de leur entourage et ajustent au plus près le choix des pièces. Ce sont des organisateurs, qui jouent un rôle de catalyseur plutôt que de conseiller car leur expertise en matière de théâtre n'est pas plus grande que celle des autres. En revanche, ils ont un effet d'entraînement sur ce groupe parce qu'ils prennent en charge la recherche de spectacles ou la réservation des places. Mais parfois ce sont d'autres qu'eux qui le font, «ça tourne » comme en témoigne une spectatrice. Ils peuvent agir sur de tout petits groupes, un conjoint, des voisins, un couple ami, ou des groupes plus importants comme les groupes d'amis qui font du théâtre une sortie régulière. Le fonctionnement est toujours le même : on écoute ce que veulent les autres, on sait ce qu'ils aiment d'habitude, tout le monde a plus ou moins les mêmes attentes de répertoire ou de style de pièce, on ne cherche pas à être audacieux mais à réussir la sortie. C'est autour de ces personnes que se nouent la plupart des réseaux qui fédèrent les spectateurs occasionnels.

À un niveau intermédiaire, on peut repérer des conseillers plus engagés, qui cherchent plus souvent à faire de nouvelles propositions à leur entourage qu'à conforter des goûts déjà constitués. Ces propositions ne sont pas forcément audacieuses, mais elles témoignent d'une expertise plus grande ou d'une information plus sophistiquée sur l'offre. La différence la plus nette avec le type précédent tient au fait qu'il s'agit d'individus qui se sentent en position légitime de conseil, parce qu'ils pensent en savoir davantage. Et qu'ils sont reconnus comme tels par ceux qu'ils conseillent. Le terme de conseiller est sans doute trop fort, même s'il est parfois utilisé dans les entretiens. Il s'agirait plutôt d'informateurs, qu'on connaît bien, et auxquels on fait appel tout en gardant une certaine liberté dans le choix.

\section{Le rôle des spectateurs relais}

On passe à un autre niveau avec les spectateurs que l'on peut qualifier de «relais », qui jouent un rôle de pédagogues et cherchent à initier d'autres spectateurs. Le statut de spectateur relais est officialisé dans certains thêâtres (voir Les groupes d'amis du Théâtre national de Chaillot, p. 10), et s'accompagne parfois d'avantages concrets (places ou abonnements gratuits) ou de gratifications symboliques (participation à des actions culturelles hors les murs, invitations aux premières, échanges

7. Certaines personnes interrogées évoquent des conversations sur le théâtre dans les salles des professeurs.

8. B. ERICKSON, "Culture, Class and Connections", American Journal of Sociology, 1996, 102, p. 217-251.

9. Seulement $8 \%$ des spectateurs du théâtre de Cavaillon disent choisir leurs pièces selon des conseils de leurs proches, contre $43 \%$ en fonction du nom du metteur en scène et $33 \%$ de celui de l'auteur ou du texte (A. DJAKOUANE, les Carrières de spectateurs..., op. cit., p. 277). La proportion est toutefois plus élevée pour le public de la Comédie-Française : $22 \%$ des spectateurs avaient entendu parler avant de la pièce qu'ils viennent de voir, dont les deux tiers par des personnes de leur entourage et de leur génération (V. BEAUdouIn, B. MARESCA, les Publics de la Comédie-Française, op. cit., p. 171174). 
très personnalisés avec les relations publiques...). Qui sont ces spectateurs relais? D'abord des enseignants, et au premier chef les professeurs de français, mais l'on peut aussi associer à cette catégorie certains des responsables de groupes d'amis particulièrement actifs, qui gèrent souvent plusieurs abonnements de groupe. Paradoxalement, les personnes dont l'activité tourne autour du théâtre (étudiants dans les filières théâtre ou comédiens amateurs par exemple) ne font pas partie de cette catégorie: les entretiens révèlent qu'elles vivent dans un milieu où tout le monde va très souvent au théâtre et qu'elles ne cherchent pas particulièrement à conseiller des connaissances qui seraient moins expertes, mais plutôt à enrichir leur répertoire et à expérimenter des pièces ou des propositions artistiques difficiles - qu'il serait de toute façon difficile de conseiller à un spectateur lambda.

Les conseillers relais sont des pratiquants très assidus, des «hyper publics», qui jouent un rôle très important dans la diffusion de l'information pour le secteur subventionné - une personne interrogée se définit comme « une petite fourmi qui permet aux théâtres de bien fonctionner». Sensibles à la reconnaissance qu'ont les théâtres pour leur prosélytisme en faveur du spectacle vivant en général, et de la programmation d'une salle en particulier, ils sont désireux d'obtenir certaines gratifications, fussent-elles seulement symboliques.

\section{AVEC QUi Aller AU THÉÂTre?}

Dans le cas du théâtre, stabiliser un réseau de pratique est très important: il ne suffit pas de recruter des compagnons pour une sortie, il faut aussi pouvoir reproduire l'expérience sans se poser chaque fois la question de l'accompagnement. C'est d'autant plus essentiel, que la sortie au théâtre est une pratique très rarement solitaire : seulement $4 \%$ de spectateurs seuls, contre $8 \%$ dans le cas des visiteurs de musée (tableau 1). On observe d'ailleurs une grande unanimité sur le fait que l'expérience théâtrale est une expérience de partage. Les rares solitaires rencontrés lors des différentes enquêtes qualitatives appartiennent à deux profils opposés : d'un côté, des spectateurs qui ne parviennent pas à recruter des accompagnants dans leur entourage ( Je sais ça surprend beaucoup parce qu'on dit : "Oh tu y vas toute seule !..” ah ben oui, oui... Faute de combattant, hein, ben j'y vais toute seule ! »). De l'autre, des hyperpratiquants (le pourcentage de solos passe d'ailleurs de $3 \%$ chez les spectateurs occasionnels à $6 \%$ chez les spectateurs assidus), qui ont souvent un lien quasi professionnel avec le spectacle vivant et que la sortie en solitaire ne dérange pas. L'enquête sur les musées de Bourdieu et de Darbel dressait d'ailleurs déjà ce constat: les amateurs de leur enquête disaient préférer les visites en solitaire ${ }^{10}$.
On peut comparer les configurations sociales des visites de musée ${ }^{11}$ et des sorties au théâtre, puisque la question était posée dans les mêmes termes dans l'enquête Pratiques culturelles des Français de 2008. Un point commun apparaît : comme pour le théâtre, c'est la sortie en couple qui est la plus répandue, suivie de très près par la visite en famille avec les enfants, alors qu'à l'inverse, la visite régulière de musée avec un groupe d'amis est nettement moins fréquente que dans le cas du théâtre. Ainsi, ce serait donc une plus forte présence des configurations sociales amicales qui caractériserait la sortie au théâtre.

Les modes de sortie au théâtre et au musée sont toutefois souvent liés : lorsque la dernière sortie au théâtre s'est faite en couple, c'est aussi le plus souvent en couple que l'on a effectué sa dernière sortie au musée. Même constat pour ceux qui sont allés au théâtre avec les amis habituels ou en famille avec des enfants mineurs : ils sont nombreux à avoir fait leur dernière visite de musée dans les mêmes configurations d'accompagnement.

Il est possible, à la lumière des résultats de la dernière enquête Pratiques culturelles des Français de 2008 relatifs à la question sur les accompagnants («Avec qui êtesvous allé(e) au théâtre ${ }^{12}$ la dernière fois ? »), de préciser le profil des publics du théâtre en fonction du réseau de sociabilité mobilisé (tableau 2, page 10).

Les sorties en couple viennent largement en tête (37\% des spectateurs), et si l'on y ajoute les sorties en famille avec des enfants mineurs (13\%), et en famille sans enfants (7\%), on constate que plus d'un spectateur sur deux va au théâtre avec des membres de sa famille. En ce qui concerne les sorties avec l'entourage amical, les sorties « spécialisées » («avec un ou plusieurs amis avec lesquels vous avez l'habitude d'aller au thêâtre ») sont plus de deux fois plus fréquentes (17\%) que les sorties avec

\section{Tableau 1 - Réseau mobilisé pour la dernière sortie au théâtre et au musée}

$\begin{array}{|lcc|} & \begin{array}{c}\text { Spectateurs } \\ \text { de théâtre }\end{array} & \begin{array}{c}\text { Visiteurs } \\ \text { de musée }\end{array} \\ \text { En couple } & 37 & 27 \\ \text { Avec des amis réguliers } & 17 & 9 \\ \text { En famille avec enfants mineurs } & 13 & 26 \\ \text { Avec d'autres amis } & 7 & 6 \\ \text { En famille sans enfants } & 7 & 7 \\ \text { En groupe (scolaire, 3e âge...) } & 7 & 8 \\ \text { Seul } & 4 & 8 \\ \text { Plusieurs réseaux } & 8 & 10 \\ \text { Total } & 100 & 100 \\ \text { Base: spectateurs de théâtre et visiteurs de musée au moins une fois au } \\ \text { cours des douze derniers mois. }\end{array}$

Source : enquête Pratiques culturelles des Français, 2008 DEPS, Ministère de la Culture et de la Communication, 2013.

10. Pierre Bourdieu et Alain Darbel, l'Amour de l'art. Les musées d'art européens et leur public, Paris, Minuit, 1966.

11. Le public du musée est plus nombreux que celui du théâtre : $30 \%$ des plus de 15 ans sont allés au moins une fois au musée au cours des douze derniers mois contre $19 \%$ pour le théâtre.

12. La question posée dans l'enquête Pratiques culturelles des Français distingue le théâtre professionnel des spectacles de rue, des spectacles amateurs, des ballets ou de l'opéra. 
d'autres types d'amis (7\%). Les sorties en groupe organisé ne concernent que $7 \%$ du public du théâtre et sont largement concentrées sur les tranches d'âge 15-25 ans. Enfin, le cumul des réseaux - par exemple en couple au sein d'un groupe amical - est relativement faible, $8 \%$ des spectateurs.

L'équilibre entre sorties familiales et sorties amicales varie avec la fréquence de la pratique. Plus on va souvent au thêâtre, plus les accompagnants se recrutent dans un réseau amical régulier et non dans l'entourage familial comme c'est le cas pour les spectateurs occasionnels. Mais ce sont probablement les différences entre les configurations de sorties masculines et féminines qui sont les plus frappantes, surtout dans le cas des sorties en couple (62\% des spectateurs déclarant être sortis en couple sont des hommes et seulement $38 \%$ des femmes) et des sorties avec des amis réguliers (pratiquées à $72 \%$ par des femmes). Il y a presque autant d'hommes que de femmes qui sont allés au théâtre en France au cours des douze derniers mois, mais leur manière de le faire diffère fortement.

\section{Les sorties en famille avec ou sans enfants}

Soulignons tout d'abord que la sortie en famille sans enfants relève d'une logique très différente de celle qui se fait avec eux. La sortie en famille sans enfants mineurs est deux fois plus fréquente parmi les habitants d'Île-deFrance que pour les Parisiens, plus fréquente aussi parmi les femmes au foyer et les célibataires sans enfants, ainsi que dans les foyers à faible revenu. On voit bien qu'il s'agit d'une sortie sur un mode exceptionnel, aux antipodes des pratiques des spectateurs parisiens les plus assidus. On note aussi qu'elle est presque deux fois plus souvent pratiquée par les femmes que les hommes.

Ces sorties en famille sans enfants recouvrent une multiplicité de situations que le terrain qualitatif n'épuise qu'en partie. Un certain nombre de personnes interrogées évoquent des visites des membres de leur famille de province qui sont l'occasion de faire une sortie au théâtre à Paris. Le choix se porte souvent sur des théâtres privés qui programment une «tête d'affiche »-ce sont des sorties rares et festives - mais on rencontre aussi des couples mère-fille qui continuent de fréquenter le théâtre à l'âge adulte, des sorties offertes à des parents âgés comme cadeau d'anniversaire, des frères et sœurs adultes qui font de la sortie au théâtre une occasion de se voir... La plus grande proportion de femmes pour cette configuration de sortie s'expliquerait donc par le fait que, dans la sortie au théâtre comme dans beaucoup d'autres domaines, ce sont les femmes qui se chargent de la gestion des liens familiaux ${ }^{13}$.
La sortie avec des enfants de moins de 18 ans ne recrute pas le même public. Elle est privilégiée par des classes moyennes faiblement diplômées qui vivent hors de la région parisienne. C'est une sortie de professions intermédiaires et d'employés ayant un diplôme inférieur au bac, et résidant dans des communes rurales ou des communes de moins de 20000 habitants. De plus, mais on s'en doutait, elle est fortement liée à la présence d'enfants mineurs dans le foyer. Là encore, c'est une modalité de sortie que les femmes prennent plus souvent en charge que les hommes (tableau 2). Plusieurs enquêtes ont déjà mis en évidence ce phénomène: la fonction éducative qui pèse largement sur les mères s'étend à la sphère des loisirs culturels ${ }^{14}$. Anne Jonchery, dans un travail sur les visites en famille au Muséum national d'histoire naturelle, montre ainsi que $43 \%$ des groupes familiaux comptent les deux parents, $38 \%$ des femmes seules avec leurs enfants et seulement $19 \%$ des pères seuls ${ }^{15}$.

Contrairement aux sorties en famille sans les enfants, qui résultent d'opportunités et de moments particuliers, on devine des logiques affirmées derrière ces sorties avec les enfants. La première consiste à se servir du théâtre comme d'un outil pour accompagner le programme scolaire (français, histoire, et même langues dans le cas des pièces surtitrées). Ce rôle est en général dévolu à l'école (les sorties en groupe concernent $82 \%$ des élèves ou étudiants) mais certains parents le prennent aussi à leur charge.

La seconde logique, celle de la socialisation à l'expérience théâtrale, a été évoquée par un grand nombre de personnes interrogées, souvent des spectateurs assidus, et leur tient visiblement à cœur. Il faut dire qu'elle pose une question de première importance, celle de la transmission d'une pratique culturelle. L'enquête réalisée par le DEPS sur la transmission des passions montre que les femmes sont deux fois plus souvent des passeuses culturelles que les hommes et que la passion pour la lecture et la musique se transmet mieux que celle pour le théâtre et les arts plastiques - ce qui n'a rien de surprenant puisque ces deux dernières activités ne s'inscrivent pas dans la quotidienneté et supposent une sortie hors du domicile ${ }^{16}$. En ce qui concerne le théâtre, l'enjeu est crucial : on sait que, dans le cas du théâtre, les sorties pendant l'enfance constituent une variable décisive dans l'acquisition de la pratique à l'âge adulte, mais le fait que ces sorties aient été effectuées en famille, avec l'école ou encore avec des amis, ne joue pas. La prescription familiale n'est donc pas plus efficace qu'une autre et l'important reste d'avoir eu une expérience du théâtre jeune, de quelque manière que ce soit ${ }^{17}$.

13. Sur la gestion du lien familial par les femmes, voir notamment Claudine AtTias Donfut, Nicole Lapierre, Martine Segalen, le Nouvel esprit de famille, Paris, Odile Jacob, 2002.

14. Voir notamment Sylvie Octobre, Christine Détrez, Pierre Mercklé, Nathalie Berthomier, l'Enfance des loisirs. Trajectoires communes et parcours individuels de la fin de l'enfance à la grande adolescence, Paris, Ministère de la Culture et de la Communication, DEPS, coll. «Questions de culture », 2010. 15. Anne JONCHERY, Michel VAN PRAËT, « Sortir en famille au musée : optimiser les négociations à l'œuvre », dans J. EDELMAN (sous la dir. de), la Place des publics. De l'usage des études et recherches par les musées, Paris, La Documentation française, 2007.

16. O. Donnat, «Transmettre une passion culturelle», Développement culturel, 2004, $\mathrm{n}^{\circ} 143$; S. OCTOBRE, « Du féminin et du masculin : genre et trajectoires culturelles », Réseaux, 2011, 168-169, p. 25-59; S. OCTOBRE, C. DÉTREZ, P. MERCKLÉ, N. BERTHOMIER, l'Enfance des loisirs..., op. cit., p. 186-190. 17. Comme le constate A. DJAKOuAne, les Carrières de spectateurs..., op. cit., p. 254-256, à partir de l'enquête sur la participation à la vie culturelle (PCV) de l'Insee, année 2000. 


\section{La sortie en couple}

La prédominance des sorties en couple, déjà constatée par d'autres travaux sur le théâtre ${ }^{18}$, $\mathrm{s}^{\text {'explique assez }}$ aisément, ne serait-ce qu'en termes d'organisation : la vie en couple suppose une bonne maîtrise de l'agenda du conjoint. Toutefois, les données de la nouvelle question intégrée dans l'enquête Pratiques culturelles des Français de 2008 permettent de montrer que la sortie en couple n'est pas du tout pratiquée avec la même intensité par les hommes et par les femmes. L'apparente préférence des hommes pour les sorties en couple commence très tôt dans la vie et s'accentue nettement après 50 ans (graphique 1). Si l'on s'intéresse uniquement aux spectateurs vivant en couple (mariage ou vie maritale), on constate que l'écart entre hommes et femmes reste très grand : $66 \%$ des spectateurs masculins vivant en couple vont au théâtre en couple, contre seulement $47 \%$ des spectatrices dans le même cas. On note aussi que ces sorties sont beaucoup plus pratiquées par les personnes en couple qui n'ont pas ou plus d'enfants de moins de 18 ans vivant chez elles.

Enfin, la sortie en couple est plus fréquente chez les spectateurs occasionnels que chez les spectateurs assidus (tableau 2). Comme pour le public du théâtre de Cavaillon, les spectateurs qui viennent au théâtre en couple font des choix de pièces moins éclectiques que les autres ${ }^{19}$. On peut donc faire l'hypothèse que la sortie en couple recrute un public moins connaisseur.

Les entretiens permettent de comprendre que, dans l'immense majorité des couples, c'est la femme qui s'occupe de l'organisation pratique de la sortie. La question a été systématiquement posée : réserver les places, prendre les billets, résoudre les questions d'ordre domestique comme la garde des enfants, toutes ces tâches incombent aux conjointes.

Les entretiens permettent de distinguer trois types de couples $^{20}$ :

- les couples où les deux conjoints partagent à la fois une appétence pour le théâtre et les mêmes goûts en matière de pièces. Ce type de couple est aussi bien représenté chez des spectateurs occasionnels du théâtre privé qui se cantonnent au registre de la comédie, que chez des couples d'enseignants qui sont au contraire des assidus du théâtre public ;

- les couples où l'un des individus - souvent la femme a une pratique ou une connaissance du théâtre très supérieure à celle de son conjoint. Dans ces couples, celui qui est amateur de théâtre effectue certaines sorties avec son conjoint et d'autres sans lui (notamment seul). Il leur faut trouver une sorte d'équilibre culturel
Graphique 1 - Sortie au théâtre en couple selon le sexe et l'âge

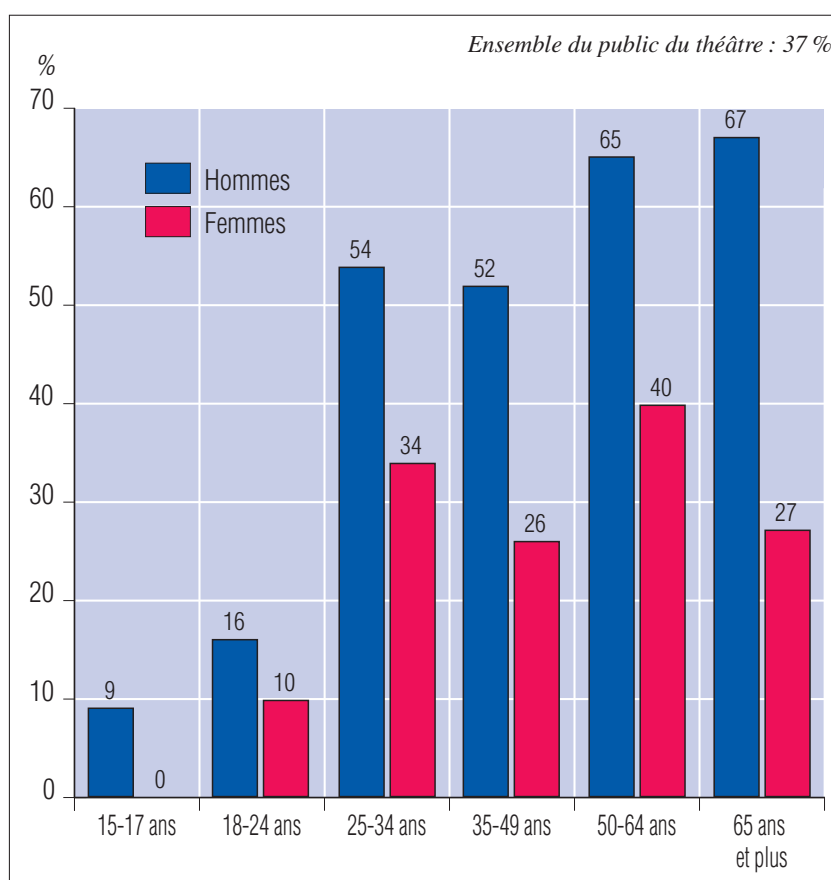

Base: individus ayant vu au moins une pièce de théâtre au cours des douze derniers mois.

Note de lecture: sur 100 hommes âgés de 65 ans et plus qui sont allés au théâtre au cours des douze derniers mois, 67 y sont allés la dernière fois en couple. Cette proportion est de 27 chez les spectatrices du même âge.

Source : DEPS, Ministère de la Culture et de la Communication, 2013.

pour les sorties faites à deux, en dosant la fréquence de celles-ci, et en évitant les expériences trop déconcertantes ;

- les couples qui maintiennent une pratique du théâtre à deux tout en surmontant certains différends quant à l'appréciation des pièces, voire de la pratique du théâtre en général, avec là encore un déséquilibre d'appétence pour le théâtre en faveur des femmes. Dans ces couples, les conjointes ne sont pas forcément des connaisseuses, mais elles aiment aller au théâtre et cherchent à entraîner leur conjoint aussi souvent que possible. Ce couple est le fruit d'un arrangement social : nous l'avons rencontré plus souvent chez des personnes habitant la province, en particulier des petites villes, où la sortie séparée est probablement, sinon moins tolérée, du moins plus visible, notamment pour une sortie au théâtre qui reste relativement exceptionnelle. On peut aussi évoquer, comme le suggère Collins, la division sexuée du travail de statut: c'est aux femmes qu'incombe le devoir de représentation publique du statut culturel de la famille ${ }^{21}$. Ce dernier type conduit à reconsidérer le pourcentage très important des hommes qui

18. J.-M. Guy, L. Mironer, les Publics du théâtre..., op. cit. A. Duakouane, les Carrières de spectateurs..., op. cit.

19. A. DJaKouAne, les Carrières de spectateurs..., op. cit., p. 318.

20. A. Duakouane (les Carrières de spectateurs..., op. cit., p. 301-302) dans son enquête sur le public du théâtre de Cavaillon en évoquait quatre : le couple complice qui partage les mêmes goûts depuis toujours, le couple où l'un des conjoints joue un rôle de mentor pour l'autre et l'initie à de nouvelles formes théâtrales, le couple où seul l'un des conjoints va au théâtre, et le couple dont les deux conjoints finissent par abandonner la pratique du théâtre faute de trouver une offre qui leur convienne à tous deux.

21. R. Collins, "Women and the Production of Status Cultures", dans M. LAmont et M. Fournier (sous la dir. de), Cultivating Differences: Symbolic Boundaries and the Making of Inequality, Chicago, Chicago University Press, 1992. 
sortent au théâtre en couple: on pouvait penser que c'était là le mode de sortie qu'ils préféraient, mais il peut aussi s'agir de sorties concédées à leur conjointe, sans goût personnel pour le théâtre. Le fait que les sorties en couple soient proportionnellement plus fréquentes chez les spectateurs occasionnels que chez les spectateurs assidus conforte cette hypothèse.

Les témoignages recueillis au sein de ce dernier type de couple laissent transparaittre une certaine inquiétude du côté des conjointes : un mauvais choix ou, pire, un désaccord sur la pièce, laisse des traces ( « Si ça lui plaît pas et que moi ça m'a plu, ben en couple c'est toujours plus intime qu'entre amis !»). Car se tromper sur les goûts de la personne dont on partage la vie a plus de conséquences, à commencer par le fait qu'elle risque d'exprimer des réticences lorsqu'il s'agira de retourner au théâtre.

\section{La sortie avec des amis réguliers}

« Il y a ça aussi, en fait, les maris sont en général pas intéressés, c'est pour ça qu'on s'y retrouve entre nanas. » Ces propos d'une Parisienne résument bien une première caractéristique des sorties avec des amis avec lesquels on sort régulièrement au théâtre : ils comptent une forte majorité de femmes, et sont même parfois exclusivement féminins ( «Surtout pas d'hommes ! on a essayé de les emmener une ou deux fois : "Hou là, là..." »).

Ce mode de sortie, nettement plus fréquent chez les spectatrices que chez les spectateurs, atteint pour cellesci des proportions particulièrement élevées entre 18 et 25 ans d'un côté, après 50 ans et surtout 65 ans de l'autre (graphique 2) : ce sont deux moments du cycle de vie qui encadrent la période probable d'installation en couple et de naissance des enfants, période durant laquelle les sorties sont moins nombreuses et la probabilité de les faire en couple plus grande. Pour les femmes âgées de moins de 25 ans, la sortie avec des amies est sans doute une manière de pallier le déficit d'accompagnateurs masculins au thêâtre : c'est en effet entre 15 et 24 ans que la différence de fréquentation du théâtre est la plus importante entre les hommes et les femmes ${ }^{22}$. Après 50 ans, le réinvestissement dans une sociabilité amicale stable pour le théâtre tient sans doute à plusieurs facteurs : le plus grand nombre de femmes seules du fait des divorces ou veuvages, mais aussi les difficultés à maintenir sur le long terme les sorties en couple au théâtre évoquées précédemment.

Deuxième caractéristique : on observe une sur-représentation des sorties avec des amis réguliers à Paris intramuros (les Parisiens représentent $10 \%$ des spectateurs de théâtre mais $18 \%$ de ce type de sorties). Pourquoi Paris, et pas Marseille ou Lyon? Pourquoi un tel écart entre Paris intra-muros et sa banlieue? On peut émettre plu-

\section{Graphique 2-Sortie au théâtre avec des amis} réguliers selon le sexe et l'âge

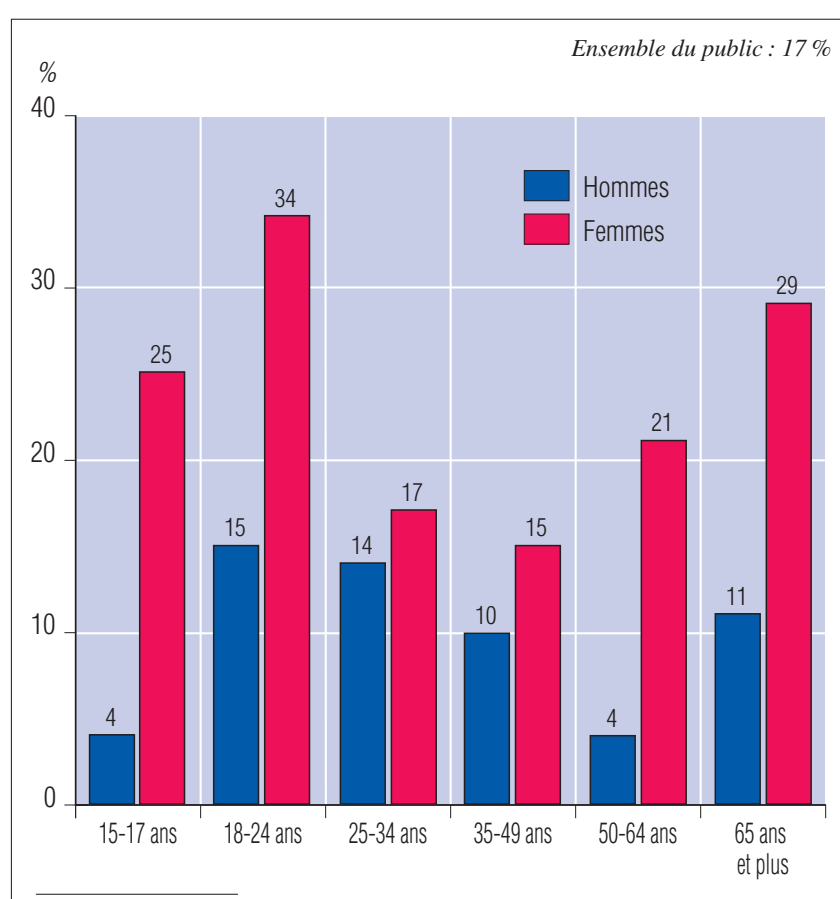

Base: individus ayant vu au moins une pièce de théâtre au cours des douze derniers mois

Note de lecture : sur 100 hommes âgés de 65 ans et plus qui sont allés au théâtre au cours des douze derniers mois, 11 y sont allés la dernière fois avec des amis ; cette proportion est de 29 chez les spectatrices du même âge.

Source : DEPS, Ministère de la Culture et de la Communication, 2013.

sieurs hypothèses. La première tient aux particularités de la capitale en matière d'offre et de consommation de théâtre ${ }^{23}$. Avec plus de deux cents théâtres, l'offre parisienne n'a pas d'équivalent, et, de fait, les Parisiens sont de bien plus grands consommateurs de théâtre que les autres : $56 \%$ de pratiquants au cours des douze derniers mois contre $19 \%$ à l'échelle de l'ensemble de la population française, dont $35 \%$ de pratiquants assidus (plus de trois sorties dans l'année) contre $6 \%$ en moyenne ${ }^{24}$. La seconde tient au nombre élevé de Parisiens qui ne vivent pas en couple. Enfin, certains avancent l'idée que la dispersion de l'habitat, les horaires de travail et les difficultés de transports dans la région parisienne distendent les liens amicaux, et que ces sorties régulières au théâtre sont un moyen de les maintenir sur une base durable, sans compter l'effet d'entraînement ( $Y$ Y'a la dynamique impulsion, ça force à sortir de chez soi »).

Cette forme de sortie concerne des groupes plus ou moins formels et étendus : dans leur forme la plus simple, ils peuvent être composés de deux personnes qui ont pris l'habitude de sortir ensemble pour voir des pièces. Au niveau le plus formel, on trouve les groupes d'amis ${ }^{25}$.

22. O. DonNaT, «La féminisation des pratiques culturelles », Développement culturel, 2005, $\mathrm{n}^{\circ} 147$.

23. De nombreux travaux ont exploré cette spécificité parisienne. Voir Bruno MARESCA, «L'intensité de la consommation culturelle, signe d'urbanité », dans O. Donnat (sous la dir. de), Regards croisés sur les pratiques culturelles, Paris, Ministère de la Culture et de la Communication, DEP, La Documentation française, coll. «Questions de culture», 2003 ; Pierre-Michel Menger, le Travail créateur. S'accomplir dans l'incertain, Paris, Le Seuil/Gallimard, 2009, p. 547-554.

24. O. Donnat, Pratiques culturelles des français à l'ère numérique, Paris, Ministère de la Culture, DEPs/La Découverte, 2009 , p. 182.

25. Pour l'enquête sur les groupes d'amis, voir l'encadré, page 10. 
La structuration d'un groupe d'amis se fait selon un principe de sociabilité par grappe : au départ, il y a toujours un noyau dur d'amis qui se connaissent très bien (anciens camarades d'études ou enseignants dans une même école par exemple), auquel viennent s'agréger au fil du temps des amis d'amis en plus ou moins grand nombre. Il y a un mélange de liens forts et de liens faibles dans la plupart des groupes : un système Facebook avant l'heure, à ceci près qu'ici il s'agit de relations en face à face. Les enquêtés décrivent cette forme de sociabilité comme un des avantages de la formule, qui permet de faire de nouvelles connaissances et de nouer des amitiés plus variées socialement («Aujourd'hui, mon cercle d'amis parisiens va et vient un peu de ce groupe de théâtre, c'est pas forcément les mêmes, ça tourne, et comme eux-mêmes ont aussi pas mal d'amis »).

Il y a différents types de responsables, comme il y a une certaine hétérogénéité dans les groupes d'amis: certains fonctionnent depuis plusieurs années sous la houlette du même responsable, d'autres sont l'objet de regroupements plus opportunistes avec une rotation des tâches d'achat des pièces. En outre, le processus de prise de décision des pièces achetées pour une saison est plus ou moins démocratique : certains groupes se réunissent et argumentent. D'autres groupes suivent plus aveuglément les choix du responsable.

La fonction de responsable de groupe comporte des contraintes : il faut trouver des dates communes, réunir les chèques, distribuer les places. Ces contraintes sont acceptées au nom d'un contrat de sociabilité passé avec les membres du groupe ( Il faut que ça reste un plaisir pour moi, hein, je suis pas une agence de vente de billet de théâtre »). De fait, la plupart des responsables ont élaboré des règles implicites. Il faut que le groupe soit soudé par des liens amicaux et que les activités de convivialité qui encadrent les spectacles soient une manifestation de ces liens. La communauté d'expérience émotionnelle entre spectateurs venus ensemble assister à un spectacle est une dimension très importante : on vit la pièce au même moment et on en parle en sortant, sans doute plus facilement qu'en couple. Mais, plus fondamentalement, la participation à un groupe permet de socialiser le risque théâtral : les moments de convivialité après le spectacle sont décrits comme une possible compensation aux pièces ratées ( Si le théâtre est pas bien, le restaurant sera bien...»).

\section{CONCLUSION}

Les résultats de cette recherche sur la sortie au théâtre suggèrent d'analyser plus avant ce qu' on pourrait appeler les conséquences sociales du déclin des pratiques de la culture cultivée, un déclin particulièrement net dans les jeunes générations, et bien plus marqué chez les hommes que chez les femmes ${ }^{26}$. La plus grande appétence des femmes pour la culture cultivée se vérifie aujourd'hui à tous les âges, dans tous les milieux sociaux et à tous les niveaux d'éducation ${ }^{27}$. La féminisation de la culture cultivée doit bien sûr être mise en relation avec les meilleures performances scolaires féminines, qui creusent, depuis les années 1980, l'écart avec leurs homologues masculins, en particulier dans les filières liées aux humanités. Dans les cohortes d'unions les plus anciennes, l'hétérogamie était liée à un niveau d'études de l'homme supérieur à celui de sa conjointe. Pour les générations les plus récentes, c'est l'inverse ${ }^{28}$.

Ces transformations ont une conséquence majeure : les femmes sont plus souvent obligées de chercher des interlocuteurs de pratiques en dehors de leur entourage familial ${ }^{29}$, comme on l'a vu ici, avec l'exemple des sorties en couple, plus difficiles à réaliser quand on est une femme.

Le cas de la sociabilité culturelle autour du livre est intéressant de ce point de vue. À niveau de lecture égal, on constate que les femmes sont plus nombreuses que les hommes à échanger des livres et à en parler. De plus, les réseaux qu'elles mobilisent pour ces échanges ou discussions sont beaucoup plus souvent situés hors de leur famille restreinte - réseaux amicaux, famille hors foyer alors que pour les hommes lecteurs, les membres du foyer constituent la première ressource ${ }^{30}$. Plusieurs travaux ont aussi été consacrés au phénomène féminin des clubs de lecture, constitués sur des bases locales et composés de femmes amatrices de lecture qui se réunissent pour discuter d'une sélection d'ouvrages. Le travail d'Élisabeth Long montre bien qu'ils permettent à la fois d'entretenir la pratique et de créer de nouveaux liens sociau ${ }^{31}$. On est donc très proche du rôle des groupes d'amis pour la sortie au théâtre. Ainsi, des réseaux de pratique qui, par le passé, puisaient largement dans les ressources culturelles familiales propres aux classes moyennes supérieures, se déploient aujourd'hui en direction de réseaux amicaux, voire même de réseaux amicaux spécialisés autour d'une pratique. Plus encore, on assiste à un phénomène de « réciprocité » : l'activité culturelle génère du lien social ${ }^{32}$.

26. O. DonNAT, «Pratiques culturelles 1973-2008. Dynamiques générationnelles et pesanteurs sociales », Culture études 2011-7; A. CHRISTIN, «Le rôle de la socialisation artistique durant l'enfance : genre et pratiques culturelles aux États-Unis », Réseaux, 2011, 29, 168-169, p. 59-86 ; O. LIZARDO, “The Puzzle of Women's Highbrow Consumption: Integrating Gender and Work into Bourdieu's Class Theory of Taste”, Poetics, $2006,34,1$, p. 1-23. 27. E. Bihagen, T. Katz-Gerro, "Culture Consumption in Sweden: the Stability of Gender Differences", Poetics, 2000, 27, p. $327-349$.

28. M. VANDERSCHELDEN, «Homogamie professionnelle et ressemblance en termes de niveau d'études: constat et évolutions au fil des cohortes », Économie et Statistique, 2006, no 398-399, p. 33-58. Le phénomène n’est pas récent, pour la période des années 1980, Van Berkel et de Graaf montrent que le nombre de couples « mixtes » (c'est-à-dire ayant un niveau d'éducation différent) a fortement crû aux Pays-Bas entre 1979 et 1987 et que le pourcentage de couples où la femme a un plus haut niveau d'éducation que son mari est passé de $10 \%$ dans la cohorte la plus âgée à $33 \%$ dans la cohorte la plus jeune. M. Van BERKEL, N.D. DE GRAAF, "Husband's and Wife's Culture Participation and their Levels of Education: a Case of Male Dominance?", Acta Sociologica, 1995, 38, 2, p. 131-149.

29. La question de l'individualisme dans la vie commune est abordée par les sociologues de la famille qui s'intéressent à la croissance des moments de vie séparés au sein des couples : voir par exemple François DE SingLY, Libres ensemble. L'individualisme dans la vie commune, Paris, Nathan, 2000.

30. F. Gire, D. PAsquier, F. Granjon, «Culture et sociabilité : les pratiques de loisir des Français », Réseaux, 2007, n $145-146$, p. $159-215$.

31. E. Long, Book Clubs: Women and the Uses of Reading in Everyday Life, Chicago, Chicago University Press, 2003.

32. O. Lizardo, "How Cultural Tastes Shape Personal Networks", American Sociological Review, 2006,71 , p. 778-807. 


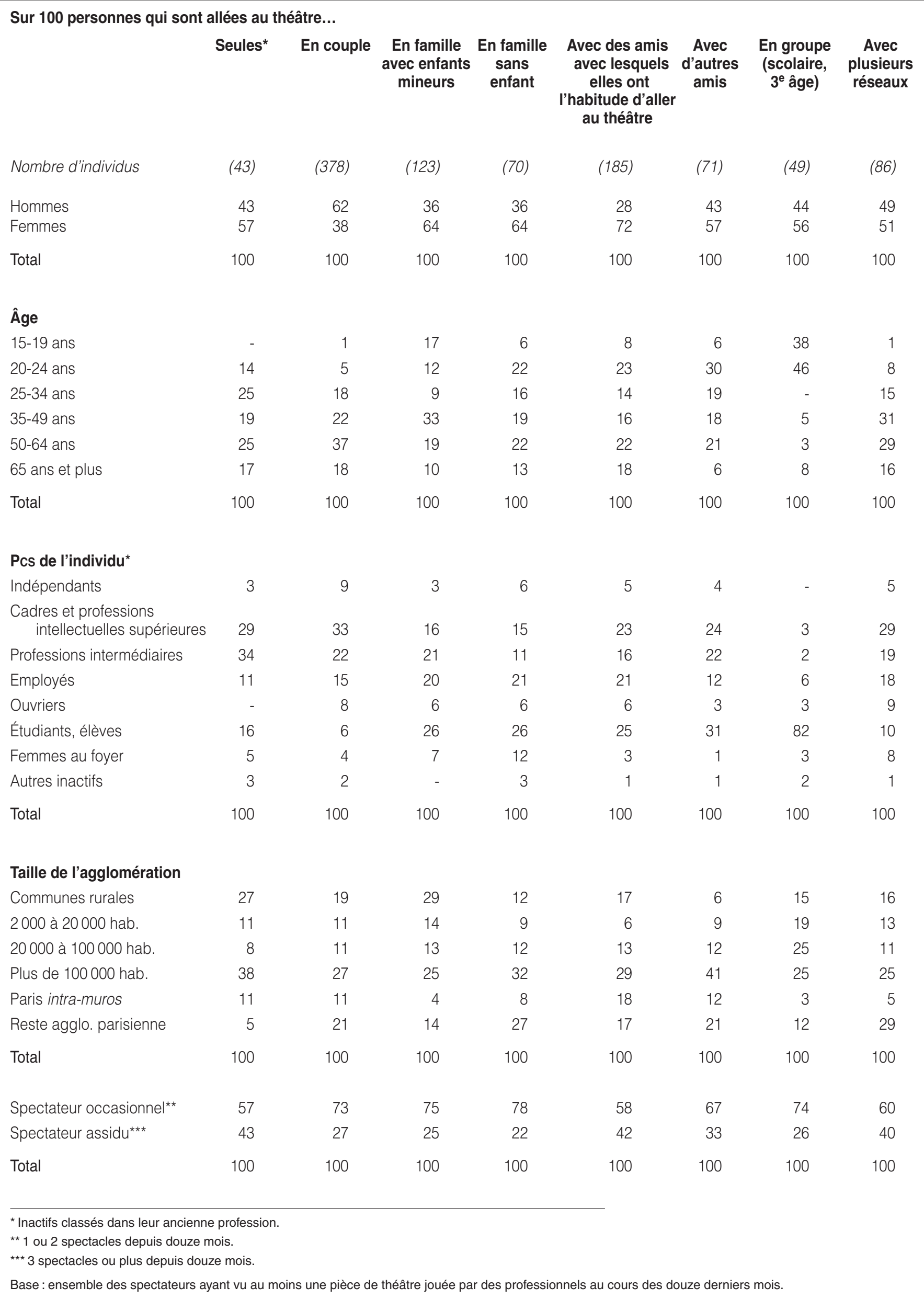




\section{Les groupes d'amis du Théâtre de Chaillot}

Le Théâtre de Chaillot comptait environ 220 groupes d'amis à l'époque de l'enquête : pour bénéficier du label, ces groupes doivent comprendre au minimum dix personnes et prendre au moins trois spectacles dans l'année. Il existe des groupes d'amis dans d'autres théâtres parisiens, mais ils sont moins nombreux (une soixantaine au Théâtre du Rond Point par exemple) et ne sont pas toujours l'objet d'un traitement de faveur particulier comme ils le sont à Chaillot. La description des groupes d'amis qui a été faite se fonde sur un cas idéal typique, où le théâtre a une politique d'entretien et de développement des groupes d'amis, et où ces derniers sont relativement stables dans le temps. Le cas de Chaillot pose à grande échelle les avantages et les inconvénients de ces sorties amicales à plusieurs et montre bien à quel point elles s'opposent à la sortie en couple - même s'il peut y avoir des couples au sein d'un groupe d'amis. L'enquête a reposé sur vingt et un entretiens semi-directifs avec des responsables de groupes d'amis, choisis au sein du fichier de Chaillot. Nous avons cherché à faire varier les âges - mais les moins de 40 ans sont peu nombreux -, les lieux de résidence et les profils des groupes (groupe ancien ou nouveau, plus ou moins nombreux, prenant beaucoup ou peu de sorties dans l'année). On peut faire plusieurs constats d'ensemble. Tout d'abord les groupes sont constitués d'une majorité de femmes, parfois même exclusivement de femmes. Ensuite, les responsables de groupe qui résident à Paris (cas de figure majoritaire à Chaillot) font souvent partie d'autres groupes d'amis dans d'autres théâtres (Théâtre de l'Odéon, Théâtre de la Ville, Théâtre du Rond-Point, pour citer les associations les plus fréquentes). Enfin, bien sûr, le fait de sortir avec un groupe d'amis à Chaillot n'empêche pas d'avoir d'autres modalités de sorties au théâtre.

\section{Éléments de méthodologie}

L'enquête s'est déroulée en 2008 et 2009, et a reposé sur plusieurs campagnes d'entretiens qualitatifs et une exploitation secondaire de l'enquête Pratiques culturelles des Français de $2008^{1}$. Les terrains ont été effectués dans l'ordre chronologique suivant: - dix entretiens préliminaires avec des responsables de services de relation au public dans différents théâtres de Paris et sa région. Ils ont permis de découvrir la pratique des "groupes d'amis ", fondés sur des réseaux amicaux informels qui prennent un abonnement ensemble et bénéficient de tarifs privilégiés ;

- enquête qualitative par entretiens avec vingt et un responsables de « groupes d'amis » du Théâtre national de Chaillot ;

- exploitation secondaire de la nouvelle question sur les accompagnants à la dernière sortie au théâtre introduite dans l'enquête Pratiques culturelles des Français de 2008 ;

- réalisation de deux monographies sur le public de province avec l'idée de saisir des situations locales spécifiques. La première s'est déroulée dans une ville de 60000 habitants de l'Ouest de la France qui ne possède qu'un seul théâtre, avec une programmation à la fois musicale et théâtrale. Dix entretiens ont été réalisés, en remontant un réseau d'individus liés par des relations interpersonnelles autour d'associations locales. La seconde monographie (20 entretiens) a été effectuée dans le Nord de la France dans une métropole urbaine où l'offre théâtrale est pléthorique, et auprès de spectateurs d'une salle ayant une programmation d'avant-garde ;

- une dernière campagne d'entretiens qualitatifs a été réalisée à partir de l'échantillon de l'enquête Pratiques culturelles des Français de 2008: trente personnes interrogées ont été tirées dans les sous-échantillons de la grande banlieue parisienne et de la région Rhône-Alpes.

1. Je remercie Florence ELOY, Fabienne GIRE, Chloé KELLER et Tomas LEGON pour leur participation au travail de terrain et de traitement des données. 


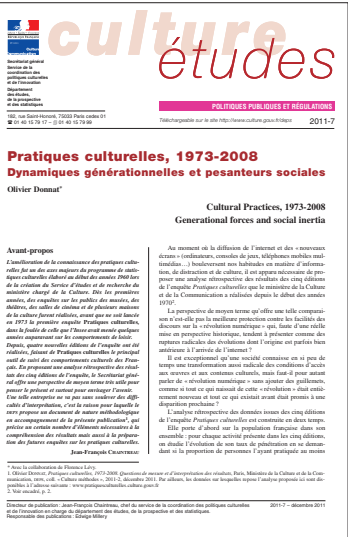

\section{CE-2011-7}

\section{Pratiques culturelles, 1973-2008 Dynamiques générationnelles et pesanteurs sociales}

\section{Olivier DONNAT}

Paris, DEPS, Ministère de la Culture

et de la Communication,

coll. "Culture études »,

décembre 2011, 36 p.

L'analyse rétrospective des cinq éditions de l'enquête Pratiques culturelles réalisées depuis le début des années 1970 met en lumière quelques grandes tendances d'évolution : l'augmentation massive de l'écoute de musique et la généralisation de la culture d'écrans, le recul de la lecture d'imprimés, l'essor des pratiques artistiques en amateur et la hausse de la fréquentation des établissements culturels. Elle souligne l'ampleur du renouvellement des pratiques culturelles, la féminisation et le vieillissement des publics, mais elle vient aussi rappeler que les dynamiques générationnelles liées à la diversification de l'offre tant publique que privée et aux profondes mutations de la société française doivent souvent composer avec les pesanteurs qui entravent le processus de démocratisation.

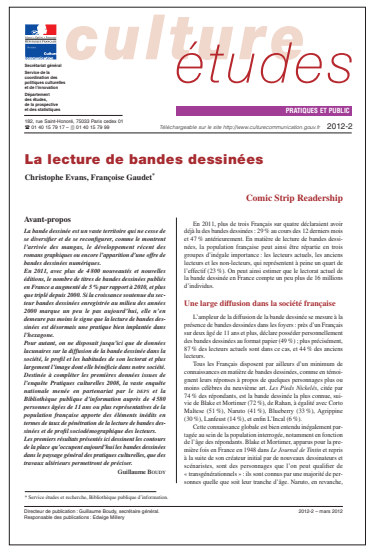

CE-2012-2

\section{La lecture de bandes dessinées}

\section{Christophe Evans, Françoise GaUdET}

Paris, DEPS, Ministère de la Culture

et de la Communication

coll. «Culture études»,

mars 2012, 8 p.

Avec plus de 16 millions de lecteurs en France, la bande dessinée, longtemps considérée comme un genre mineur, bénéficie désormais d'une large diffusion dans la société française : plus de trois Français sur quatre déclarent avoir déjà lu des bandes dessinées, dont plus d'un quart (29\%) au cours des douze derniers mois. La production éditoriale a bénéficié d'un essor sans précédent depuis les années 1900, le nombre de titres édités est en constante progression, tandis que les genres, au sein de la bande dessinée, se diversifient : aux traditionnels albums se sont ajoutés, ces dernières années, les mangas, romans graphiques, comics... L'étude apporte des éléments de caractérisation du lectorat de bandes dessinées, majoritairement masculin, plutôt jeune, par ailleurs lecteur de livres et amateur de sorties culturelles, et repère des caractéristiques de lecture propres à cette pratique, comme la relecture.

Olivier Donnat

LES PRATIQUES CULTURELLES DES FRANÇAIS A LEERE NUMÉRIOUE ENQUETE 2008

288 p., $20 €$

ISBN 978-2-7071-5800-0

9

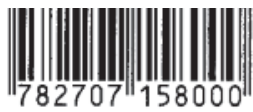

\section{Olivier Donnat}

\section{Les pratiques culturelles des Français à l'ère numérique Enquête 2008}

Depuis les années 1970, l'enquête Pratiques culturelles du ministère de la Culture et de la Communication constitue le principal baromètre des comportements des Français dans le domaine de la culture et des médias. Les résultats de 2008 révèlent, plus de dix ans après ceux de 1997, l'ampleur des effets d'une décennie de mutations induites par l'essor de la culture numérique et de l'internet.

Au moment où plus de la moitié des Français disposent chez eux d'une connexion à haut débit, où plus d'un tiers utilisent l'internet quotidiennement à des fins personnelles, comment les nouvelles formes d'accès en ligne à la culture s'articulent-elles avec la consommation des anciens médias (télévision, radio, presse écrite) et avec les pratiques culturelles traditionnelles?

Comment se portent la lecture de livres, l'écoute de musique ou la pratique en amateur d'activités artistiques?

La fréquentation des salles de cinéma, des théâtres ou des salles de concert a-t-elle baissé ou augmenté et le profil de leurs publics a-t-il évolué ?

Le présent ouvrage restitue les résultats sectoriels de l'enquête dans le domaine de la télévision, de la musique, du livre et de la presse, des sorties et visites culturelles et des pratiques en amateur, en soulignant chaque fois les permanences mais aussi les lignes de rupture qui se dessinent sous la poussée d'une culture numérique déjà très présente dans le quotidien des jeunes générations.

Les résultats complets de l'enquête sont consultables sur www.pratiquesculturelles.culture.gouv.fr 


\section{RÉSUMÉ}

$\mathrm{Si}$, du point de vue du public, le théâtre est une rencontre avec une œuvre, un metteur en scène et des comédiens, cette expérience s'accompagne de formes de sociabilité liées à la sortie. Comment choisit-on les pièces, sur la base de quels conseils et avec qui va-t-on au théâtre ? Si près d'un Français sur cinq va au théâtre au moins une fois par an, seuls $4 \%$ effectuent cette sortie seuls. Deux formes d'accompagnement prévalent : la sortie en couple et celle avec des amis avec lesquels se pratiquent des sorties au théâtre régulières. La première est beaucoup plus répandue chez les hommes, et la seconde recrute une forte majorité de femmes.

L'enquête s'appuie sur une exploitation secondaire de la dernière édition de Pratiques culturelles des Français (2008) et d'une série d'entretiens menés auprès de spectateurs de différents théâtres à Paris et en province; elle analyse les différentes formes de sociabilités associées à la sortie au théâtre. Elle confirme, dans le cas de la fréquentation du théâtre, la féminisation de la culture et met en évidence le rôle que jouent les femmes dans l'organisation des sorties.

\section{ABSTRACT}

From the audience's point of view, going to the theatre is an encounter with an art work, a direction and acting. But, this experience goes together with certain forms of sociability relating to theatre outings. How do people choose which play to see, whose advice do they follow and with whom do they go to the theatre? Although almost one in five French people go to the theatre at least once a year, only $4 \%$ do so alone. There are two major ways in which people attend with others: going either as a couple or as a group of friends who regularly organise theatre trips together. The former is far more common amongst men, whilst the latter category sees a much greater prevalence of women.

This study is based on secondary analysis of the last edition of Pratiques culturelles des Français (2008) and on a series of interviews with audience members of various theatres within Paris and the provinces; it analyses the various forms of sociability associated with theatre outings. In the case of theatre visits, it confirms the feminisation of the culture and reveals the role which women play in organising these outings.

\section{Tous les documents publiés par le DEPS sont téléchargeables sur http://www.culturecommunication.gouv.fr/Etudes-et-statistiques et sur www.cairn.info}

Pour recevoir régulièrement les publications du DEPS et pour toute demande d'information:

contact.deps@culture.gouv.fr 